\title{
EEA stapler offers hemostasis advantage over PPH
}

Published online: 01 August 2012

(C) Springer Healthcare 2012

MedWire News: The EEA stapler (Covidien, Dublin, Ireland) may have benefits over the PPH stapler (Ethicon EndoSurgery, Cincinnati, Ohio, USA) for circular stapled hemorrhoidopexy (SH), the results of the first head-to-head study of the devices show.

"The EEA stapler shows better haemostatic performance compared with PPH staplers allowing the removal of a larger area of rectal mucosa in the treatment of degree III haemorrhoids," say Donato Altomare (University of Aldo Moro, Bari, Italy) and colleagues.

The study included 135 patients with III degree hemorrhoids who were randomly assigned to receive treatment with the $\mathrm{PPH} 03$ or 01 stapler or the $33 \mathrm{~mm}$ EEA stapler.

The EEA stapler is larger than the PPH staplers and has a detachable anvil with three anchor points. In most patients, the middle hole of the detachable anvil was used to anchor the purse string, with only four patients requiring the third hole.

The authors, reporting in Colorectal Disease, found that the mean area of the resected mucosa in the EEA group was $33.75 \mathrm{~cm}^{2}$ compared with $28.05 \mathrm{~cm}^{2}$ in the PPH group.

Furthermore, fewer overstitches were required to achieve hemostasis following stapling in the EEA group, who required a median of one stitch compared with three in the PPH group.

The authors also note that while two patients developed moderate postoperative bleeding in the PPH group, no patients did in the EEA group. They say this supports the notion that EEA results in improved hemostasis.

SH using the PPH stapler is a commonly used technique for the treatment of III degree hemorrhoids. However, while it offers benefits over traditional surgical approaches, such as faster recovery and less postoperative pain, postoperative bleeding remains a concern.

Use of the PPH stapler is also associated with a high level of recurrence, with half of SH patients requiring further surgery. The authors therefore intend to conduct a long-term follow up of these patients to determine any difference in recurrence rates over time.

By Kirsty Oswald, MedWire Reporter

\section{Reference}

Colorectal Dis 2012; Advance online publication 